Revista

Actualidades Investigativas

en Educación

\title{
La sintaxis en la competencia discursiva del estudiantado universitario de las áreas no humanísticas: Una propuesta pedagógica
}

The syntax in the discursive competence of university students in nonhumanistic areas: a pedagogical proposal

Volumen 19, Número 2

Mayo-Agosto

pp. 1-22

Desiderio José Evangelista Huari Evelyn Katty Evangelista Padilla

\section{Cite este documento así}

Evangelista Huari, Desiderio José y Evangelista Padilla, Evelyn Katty. (2019). La sintaxis en la competencia discursiva del estudiantado universitario de las áreas no humanísticas: Una propuesta pedagógica. Revista Actualidades Investigativas en Educación, 19(2), 1-22. Doi. 10.15517/aie.v19i2.36902 


\title{
La sintaxis en la competencia discursiva del estudiantado universitario de las áreas no humanísticas: Una propuesta pedagógica
}

The syntax in the discursive competence of university students in non-humanistic areas: a pedagogical proposal

\author{
Desiderio José Evangelista Huari ${ }^{1}$ \\ Evelyn Katty Evangelista Padilla²
}

\begin{abstract}
Resumen: Una de las actividades recurrentes que enfrenta la población estudiantil durante su permanencia en la universidad es la composición de textos académicos. Para ello, dicha población debe contar con una competencia discursiva desarrollada. Sin embargo, quienes pertenecen a las áreas no humanísticas presentan muchas deficiencias cuando redactan. Por esa razón, realizamos una investigación cuasi experimental y de interpretación cualitativa. Nuestro objetivo es mostrar la eficacia del uso de una sintaxis básica de la lengua española en el proceso de aprendizaje $y$, de este modo, logren redactar textos coherentes y cohesionados. Para este efecto, seleccionamos una muestra de 50 discentes pertenecientes a las áreas de Ingeniería y de Arquitectura de la Universidad Nacional de Ingeniería (Perú). De esta muestra, 25 estudiantes de la especialidad de Ingeniería Económica forman el grupo de control y 25 integrantes de la especialidad de Arquitectura constituyen el grupo cuasi experimental. Después de tomar una prueba pretest a toda la muestra, aplicamos al grupo cuasi experimental la estrategia metodológica mencionada. Esta estrategia consiste en organizar textos breves mediante el uso de una sintaxis básica. Además, las secuencias oracionales deben enlazarse mediante mecanismos de cohesión como la repetición, la sustitución y la elisión para controlar la progresión temática. Concluida la aplicación de la estrategia propuesta, tomamos una evaluación de salida (postest) para conocer los resultados obtenidos. Las conclusiones que se incluyen en este artículo nos permiten corroborar la eficacia de esta estrategia para afianzar la competencia discursiva de la persona discente.
\end{abstract}

Palabras clave: discurso, sintaxis, estudiantes universitarios, arquitectura, ingeniería

\begin{abstract}
One of the recurring activities faced by the student population during their stay at the university is the composition of academic texts. For this, this population must have a developed discursive competence. However, those who belong to the non-humanistic areas have many deficiencies when they write. For that reason, we conducted a quasi-experimental research and qualitative interpretation. Our objective is to show the effectiveness of the use of a basic syntax of the Spanish language in the learning process and, in this way, to write coherent and cohesive texts. For this purpose, we selected a sample of 50 students belonging to the Engineering and Architecture areas of the National University of Engineering (Peru). Of this sample, 25 students of the specialty of Economic Engineering form the control group and 25 members of the specialty of Architecture constitute the quasi-experimental group. After taking a pretest test to the whole sample, we applied to the quasi-experimental group the aforementioned methodological strategy. This strategy consists of organizing short texts through the use of a basic syntax. In addition, sentence sequences should be linked through cohesion mechanisms such as repetition, substitution and elision to control thematic progression. Once the application of the proposed strategy is completed, we take an exit evaluation (posttest) to know the results obtained. The conclusions included in this article allow us to corroborate the effectiveness of this strategy to strengthen the discursive competence of the student.
\end{abstract}

Keywords: speech, syntax, university students, architecture, engineering

\footnotetext{
${ }^{1}$ Docente e Investigador de la Universidad Nacional Mayor de San Marcos, Lima, Perú. Dirección electrónica: devangelistah@unmsm.edu.pe

2 Docente de la Universidad Nacional Mayor de San Marcos, Lima, Perú. Dirección electrónica: eveykatty@gmail.com
}

Artículo recibido: 26 de julio, 2018

Enviado a corrección: 25 de octubre, 2018

Aprobado: 26 de noviembre, 2018 


\section{Introducción}

En el área de la redacción académica, ¿qué enseñar?, ¿para qué enseñar?, ¿cómo enseñar? son preguntas recurrentes que se formulan quienes tienen a cargo esta clase de asignaturas en la universidad. La primera interrogante se refiere a los tópicos que se imparten en esta asignatura; la segunda guarda relación con los objetivos por los cuales se implementan estos cursos, y la última tiene que ver con las estrategias a las que se recurren para afianzar la competencia textual y discursiva de la población estudiantil de este nivel de estudios.

Referente a la última interrogante, esta guarda concordancia con la redacción (o alfabetización) académica, la cual viene afianzándose en las últimas décadas en el contexto universitario. De acuerdo con Carlino (2005, p. 6), la alfabetización académica hace referencia al "conjunto de nociones y estrategias necesarias para participar en la cultura discursiva de las disciplinas así como en las actividades de producción y análisis de textos requeridas para aprender en la universidad". En nuestra opinión, el aprendizaje de estas estrategias a las que se refiere la autora está relacionado con los avances de disciplinas como la Lingüística del texto, la Lingüística Cognitiva, la Pragmática, la Psicolingüística, las estrategias pedagógicas, entre otras.

Desde luego, a pesar del desarrollo significativo de estas áreas, no ha ocurrido igual con las estrategias de aprendizaje para reforzar la competencia discursiva del estudiantado. Esto se advierte al observar el contenido de los manuales de enseñanza. Tampoco, las estrategias metodológicas responden a las características que exige la redacción académica. Esta situación se torna aún más crítica en contextos donde la población estudiantil no pertenece a las áreas humanísticas. Dicha comunidad estudiantil, a pesar de haber cursado asignaturas relacionadas con esta materia, no está capacitada plenamente para componer textos académicos que cuenten con coherencia y cohesión pertinentes. Esta realidad es preocupante, pues, a nuestro juicio, una de las causas está relacionada con el uso de estrategias metodológicas no apropiadas para tal efecto. Al respecto, Gutiérrez (2002, p. 299) precisa lo siguiente: "No conviene introducir metodologías abstrusas y difíciles de comprender tanto para los profesores como para los profesores". Como vemos, la advertencia de este autor es patente respecto de las estrategias de aprendizaje a las que recurrimos para reforzar la habilidad escrita del discente. 
Precisamente, en el presente artículo, damos a conocer los resultados de una estrategia didáctica de aprendizaje que hemos aplicado a una muestra de estudiantes de las áreas mencionadas. Esta estrategia consiste en el uso de unos módulos de sintaxis básica de la lengua española para afianzar el manejo de los mecanismos de cohesión en la redacción. En este sentido, la investigación que realizamos es de carácter cuasi experimental.

En el contexto universitario, la competencia discursiva del estudiantado está íntimamente relacionada con la producción de diferentes escritos de carácter académico. Para ese efecto, la comunidad estudiantil debe utilizar discursos altamente especializados (Cassany y Morales, 2008, p. 1). Vale decir, debe hacer uso de su competencia discursiva para llevar a cabo actividades relacionadas con la composición de textos. Por ejemplo, tiene que desarrollar pruebas escritas, preparar resúmenes, elaborar ensayos de las asignaturas que estudian, redactar informes técnicos y de laboratorio, describir la estructura de una maqueta, elaborar trabajos monográficos, componer artículos para participar en algún evento académico, entre otros.

Ahora bien, si la actividad principal de la población estudiantil universitaria está íntimamente ligada a la producción y redacción de diferentes tipos de textos, entonces:

- ¿Con qué nivel de competencia discursiva cuenta la población estudiantil universitaria que cursa los primeros años de estudios de las áreas de Arquitectura y de Ingeniería?

- ¿Cómo el uso planificado de una sintaxis básica de la lengua española le permite a esta población componer textos que cuenten con cohesión y coherencia adecuadas?

- ¿En qué medida el uso controlado de los elementos discursivos como la repetición, la sustitución y la elisión les permite redactar materiales escritos adecuados y pertinentes?

Para responder estas interrogantes, nos planteamos los siguientes objetivos:

- Identificar los niveles de competencia discursiva que posee la población estudiantil universitaria perteneciente a las áreas de Arquitectura e Ingeniería de los primeros años.

- Poner a prueba una estrategia metodológica consistente en el uso de la sintaxis básica de la lengua española para afianzar la cohesión textual en la redacción académica de la población estudiantil universitaria de las áreas mencionadas. 
- Explicar la función que cumplen la repetición, la sustitución y la elisión como mecanismos de cohesión textual en la organización de las ideas en la redacción académica de las personas discentes pertenecientes a estas áreas.

Así pues, el propósito que nos fijamos en el estudio realizado es comprobar la eficacia que brinda el manejo adecuado de una sintaxis básica y el uso planificado de los elementos cohesivos como la repetición, la sustitución y la elisión para componer textos coherentes y cohesionados.

\subsection{Base teórica de la investigación}

Actualmente, la población estudiantil universitaria demanda de estrategias de aprendizaje que le permitan reforzar su competencia discursiva y textual. En concordancia con esta idea, el presente trabajo se sustenta en las áreas de la Sintaxis, la Lingüística del texto y ambas disciplinas relacionadas con la redacción académica. A continuación, precisamos algunos puntos relevantes relacionados con estas áreas.

Respecto a la primera, como señala Bosque y Gutiérrez (2009, p. 11), "La sintaxis es la parte de la gramática que estudia la forma en que se combinan las palabras y los significados a los que dan lugar esas combinaciones". Así, la sintaxis nos permite coordinar y unir palabras para formar oraciones y expresar conceptos. En este sentido, la sintaxis no puede limitarse a distinguir la estructura de las oraciones bien formadas de otras que no lo están; antes bien, debe incluir la noción de significado para dar cuenta de dichas estructuras bien formadas.

De acuerdo con los autores citados, diremos que, al establecer la relación existente entre estructura y significado, "el significado de una determinada estructura es una función de las partes que la componen y de la forma en que se combinan" (Bosque y Gutiérrez, 2009, p. 14). Así, si queremos determinar el significado de una estructura $X$ que incluye cuatro elementos ( $A, B, C$ y $D)$; entonces, el significado de $X$ deriva de la significación de $A$, $B, C$ y $D$, y la función sintáctica que cumple $A, B, C$ y $D$ en $X$. Desde luego, en la composición escrita, esta secuencia no siempre es fija, sino que también puede variar siempre y cuando esta nueva secuencia sea agramatical y aceptable. Así, podemos generar una secuencia como: 
El sindicato de esa compañía demanda al gobierno central la solución a sus problemas.

Esta expresión es plenamente gramatical y semánticamente plausible, pero no podemos construir expresiones como:

${ }^{*} A$ sus problemas demanda, al gobierno central la solución el sindicato de esa compañía.

En este caso, la expresión no es gramaticalmente pertinente, tampoco es semánticamente plausible. Precisamente, como plantean Bosque y Gutiérrez (2009, p. 17), "construir una secuencia de palabras no equivale a superponer linealmente esquemas sintácticos, sino más bien a percibir e integrar una compleja red de relaciones". Para este efecto, resulta muy importante conocer la palabra y sus rasgos combinatorios a fin de organizar secuencias sintácticas e integrar dichas palabras en una unidad mayor que es la oración. Como manifiestan Bosque y Gutiérrez (2009, p. 24), "si las palabras son conjunto de rasgos (es decir, propiedades gramaticales), cada una de esas propiedades explicará una parte de su comportamiento". Es decir, siguiendo a estos autores, diremos que los elementos esenciales de la sintaxis no son las clases de oraciones, sino las propiedades gramaticales de las palabras.

Como se advierte, la ubicación de las palabras y la función sintáctica que estas cumplen resultan fundamentales para organizar las ideas en la composición de textos. Al respecto, según Bosque y Gutiérrez (2009, p. 25), "Las posiciones que ocupan las palabras constituyen un aspecto fundamental de la sintaxis". Es decir, si nosotros somos conscientes de la función sintáctica que desempeñan las palabras dentro de la oración, podemos recurrir a estas estrategias para organizar nuestras ideas en el proceso de la textualización.

Desde luego, actualmente hay muchos enfoques lingüísticos sobre el estudio de la sintaxis que no detallaremos en este artículo. Para nuestro propósito, bastará con fijar los conceptos básicos para explicar la eficacia de la sintaxis como estrategia metodológica en la composición de textos escritos.

En cuanto a la Lingüística del texto, esta disciplina tiene como objeto de estudio el texto. Ahora bien, ¿qué es un texto? Al respecto, hay muchas definiciones. Una definición que resulta más integral para los propósitos de este trabajo es la que presenta Bernárdez (1982). Según este autor: 
'Texto' es la unidad lingüística comunicativa fundamental, producto de la actividad verbal humana, que posee siempre carácter social; está caracterizado por su cierre semántico y comunicativo, así como por su coherencia profunda y superficial, debida a la intención (comunicativa) del hablante de crear un texto íntegro, y a su estructuración mediante dos conjuntos de reglas: las propias del nivel textual y las del sistema de la lengua. (p. 85)

De esta definición, podemos especificar el texto como una unidad de carácter

- Comunicativo. Es decir, como una actividad lingüística mediante la cual comunicamos significados.

- Social. Vale decir, como un instrumento fundamental de interacción social.

- Pragmático. Esto es, una intención comunicativa y en un contexto específico.

- Coherente. A saber, el conjunto de proposiciones que forma un significado global.

- Cohesionado. O sea, las estructuras sintácticas se ligan mediante mecanismos de cohesión.

Así pues, como plantean Calsamiglia y Tusón (2016, p. 3), el texto "está constituido por elementos verbales combinados, que forman una unidad comunicativa, intencional y completa". En este sentido, la Lingüística del texto no solo estudia la estructura gramatical de una lengua, sino que concibe el texto como un producto intencional, donde la lengua presenta una enorme heterogeneidad. Según Coseriu (2007, p. 195), desde el punto de vista funcional, las lenguas históricas nunca son completamente homogéneas. En cada lengua, podemos advertir diferencias diatópicas, diastráticas, diafásicas y diacrónicas. Estas diferencias son aún más específicas y complejas en cada texto concreto.

Si consideramos que el texto viene a ser una unidad mayor y más compleja que una oración gramatical; entonces, debemos tener en cuenta las propiedades que caracterizan a esta unidad. Así como entre las propiedades de la oración gramatical destacan la gramaticalidad y la semanticidad; del mismo modo, el texto posee reglas de textualidad como la cohesión, la coherencia, la intencionalidad, la informatividad, la intertextualidad, la situacionalidad y la adecuación (Beaugrande y Dressler, 1997).

Como vemos, el texto es una unidad supraoracional que posee sus propias propiedades y, gracias a ellas, se constituye como unidad autónoma. Entre estas 
propiedades, destacan la coherencia y la cohesión. La primera controla la progresión temática o de contenido tanto a nivel lineal como global. La segunda organiza las secuencias gramaticales, para cuyo efecto intervienen los mecanismos de cohesión como las repeticiones, las sustituciones, las elisiones, las proformas, las deixis, los tiempos verbales, las paráfrasis, los conectores, los marcadores textuales, entre otros. El siguiente texto nos permite advertir algunas de estas propiedades:

José fue al centro de la ciudad. Entró a una tienda de bicicletas y compró una bicicleta nueva. La llevó a su casa y después la guardó en el patio interior.

Aquí, los elementos resaltados constituyen los mecanismos de cohesión. Destacan, por ejemplo, la repetición (bicicleta), la sustitución (la) y las elisiones ( $\phi$ entró, $\phi$ compró, $\phi$ llevó). Desde luego, la construcción de un texto no debe verse tampoco como una actividad mecánica, sino como un proceso consciente, planificado y estratégico. Al respecto, Bernárdez (1995, p. 161) nos ilustra con el siguiente planteamiento:

Los procesos de construcción y comprensión de un texto son equivalentes a procesos de resolución de problemas: $\mathrm{P}$ debe solucionar el problema de conseguir que Mp sea adecuadamente transmitido a $P$ por medio de $\mathrm{Tr}$, i,e. tiene que construir un $\mathrm{Tp}$ que garantice el acceso de $\mathrm{R}$ a $\mathrm{Mp} ; \mathrm{R}$, por su parte, tendrá que conseguir que lo que él entiende a partir de $\mathrm{Tr}$, i.e. Mr, tenga una elevada probabilidad de coincidir con Mp.

Si bien este planteamiento puede ser discutible, de lo que estamos persuadidos es de que, para construir un texto de carácter formal y académico, debemos contar con una competencia discursiva desarrollada. Obviamente, en esta sección nuestro propósito no es estudiar el texto en todas sus particularidades, sino referirnos a sus propiedades para explicar el proceso de la textualización.

Ahora bien, ¿cómo relacionamos los estudios de la sintaxis y del texto con la redacción académica? Para explicar al respecto, a continuación nos vamos a referir brevemente a esta última. Como explicita Van Dijk (1983, p. 22), una secuencia discursiva "no sólo se ve influida por el conocimiento o las intenciones del individuo o por las funciones del texto, sino que también los grupos, instituciones y clases se comunican colectivamente o 'a través' de sus miembros mediante la producción de textos”. En estos casos, los textos académicos comparten un rasgo común: utilizan una variedad formal de una lengua. Sin 
embargo, como precisan Regueiro y Sáez (2015, p. 22), también "no todo texto académico es científico, ni todo texto científico se desarrolla en el ámbito académico; pero el lenguaje de la ciencia y el académico comparten formas, criterios y rasgos que responden a una ya larga tradición cultural". Así pues, cuando hacemos referencia a la redacción académica es necesario tomar en cuenta estas distinciones.

Naturalmente, como precisa Carlino (2005, p. 18), "la lectura y escritura exigidas en el nivel superior se aprenden en ocasión de enfrentar las prácticas de producción discursiva y consulta de textos propias de cada materia". Para este efecto, la población estudiantil debe ir familiarizándose con dichas prácticas discursivas. Al respecto, Calsamiglia y Tusón (2016, p. 65) nos puntualizan diciendo que "en cada ámbito profesional se generan actividades escritas con valor funcional, etiquetadas socialmente". Concordante con este planteamiento, debemos señalar que actualmente hay un interés primordial para afianzar la competencia discursiva de la persona discente. Desde luego, en otra publicación, Carlino (2013) sostiene que "escribir y leer en cierto contexto, y reflexionar sobre ello, sirve como experiencia. Mas no forja 'la' capacidad de hacerlo de una vez para siempre y en solitario". Es decir, saber escribir es como practicar un deporte. Cuanto más practiquemos una disciplina deportiva, lograremos mayor competencia en esta actividad.

Precisamente, cuando estudiamos la competencia discursiva de la población estudiantil de las áreas no humanísticas, nuestro propósito no solo es advertir los rasgos discursivos que caracterizan sus escritos, sino también buscar alternativas que permitan afianzar dicha competencia.

\section{Método}

Para llevar a cabo este estudio, optamos por una investigación cuasi experimental. Decimos "cuasi experimental", debido a que nos permite poner a prueba un modelo pedagógico consistente en el uso de la sintaxis básica de la lengua española para componer textos escritos coherentes y cohesivos. Para ello, elaboramos unos módulos de sintaxis básica de esta lengua para afianzar la competencia discursiva de quienes integraran el grupo cuasi experimental. 


\subsection{Muestra}

Para aplicar la estrategia metodológica mencionada, seleccionamos una muestra de 50 estudiantes pertenecientes a las áreas de Arquitectura e Ingeniería de la Universidad Nacional de Ingeniería (Lima, Perú). La Tabla 1 permite especificar las características de la muestra seleccionada.

Tabla 1

Características del grupo cuasi experimental y del grupo de control. Universidad Nacional de Ingeniería, Lima, Perú.

\begin{tabular}{|c|c|}
\hline Grupo cuasi experimental & \\
\hline $\begin{array}{l}\text { - } 25 \text { estudiantes de la especialidad de } \\
\text { Arquitectura. } \\
\text { - Grupo mixto, conformado por varones y mujeres. } \\
\text { - Seleccionado aleatoriamente. } \\
\text { - Perteneciente al primer ciclo académico. }\end{array}$ & $\begin{array}{l}\text { diantes de la especialidad de Ingeniería } \\
\text { ica. } \\
\text { ixto, conformado por varones y mujeres. } \\
\text { nado aleatoriamente. }\end{array}$ \\
\hline
\end{tabular}
Fuente: Elaboración propia (2017).

\subsection{Actividades previas a la fase cuasi experimental}

Al inicio del periodo académico 2017-II (setiembre - diciembre), aplicamos una prueba de entrada (pretest) al grupo de control y al grupo experimental. Esta prueba consistió en la redacción de un texto académico. El texto versó sobre el tema: La explotación minera, los relaves mineros y la contaminación ambiental. La extensión del texto fue aproximadamente de unas 300 palabras y el tiempo requerido para su elaboración fue de 120 minutos, tiempo tanto para escribir el borrador como para redactar la versión final. Los resultados de la prueba se resumen en las siguientes líneas:

1. En los textos, no se advierten estrategias de redacción que prioricen algún aspecto específico de la textualización.

2. La extensión de las oraciones, generalmente, es extensa. La mayoría de los textos posee entre 6 a 9 oraciones, cada una de las cuales contiene más de 30 palabras. Incluso en varias oraciones el número de palabras asciende a 85 o 90.

3. No hay marcas de cohesión textual utilizadas estratégicamente para enlazar las oraciones y así poder mantener la progresión temática en el texto.

4. La frecuencia del relativo que es alta, pues este relacionante permite expandir las cláusulas de forma recursiva haciendo que las oraciones resulten complejas.

5. El uso de los signos de puntuación es elemental. Se recurre, sobremanera, al uso de las comas para entrelazar las frases. Esta estrategia conlleva a que el texto resulte confuso y enrevesado. 
6. Respecto a la coherencia, si bien es cierto que los textos son comprensibles en alguna medida; sin embargo, muchos resultan enrevesados e imprecisos.

Así pues, esta prueba nos ha permitido conocer el estado de la cuestión respecto del nivel de competencia discursiva que poseen las personas integrantes de la muestra seleccionada.

\subsection{Actividades específicas realizadas en la fase cuasi experimental}

1. Después de tomar la prueba de entrada, iniciamos el trabajo cuasi experimental con el grupo seleccionado. Estas actividades consistieron en la redacción de textos académicos breves.

2. En el desarrollo de estas actividades, se incidió, sobremanera, en la composición de textos mediante el uso de oraciones breves y estableciendo claramente la función de los mecanismos de cohesión como la repetición, la sustitución y las elisiones.

3. En cada una de las sesiones pedagógicas y con la participación directa de los integrantes del grupo, se comentó la redacción de algunos escritos. Para contrastar estos escritos, se mostraron textos prototípicos referidos a la Física y a la Arquitectura a fin de advertir sus características sintácticas.

4. El trabajo concluyó con una prueba de salida (postest) tomada a ambos grupos. Dicha prueba consistió en la redacción de un texto breve, aproximadamente de trescientas palabras y en un tiempo de 120 minutos. Para ello, se les asignó un tema análogo a la prueba de entrada, cuyo título fue La contaminación ambiental.

5. La prueba final fue analizada sobre la base de valoraciones cualitativas. Respecto a la cohesión y la coherencia, tuvimos en cuenta tanto la consistencia de las secuencias sintácticas como la función que cumplen los mecanismos de cohesión (repetición, sustitución y elisión), pues estos elementos posibilitan la progresión temática.

\section{Descripción, análisis y discusión de los resultados}

Para analizar los resultados de la prueba de salida, elaboramos la Tabla 2, en la que especificamos los siguiente:

- En primer lugar, se precisa el número de integrantes de la muestra (de 1 a 25).

- En la comuna I, visualizamos el uso de las repeticiones.

- La columna II representa las sustituciones. 
- La columna III especifica las elisiones.

Cada columna, a su vez, detalla el valor de los mecanismos de cohesión como muy bueno (MB), bueno (B), regular (R), deficiente (D), muy deficiente (MD). Además, estos valores asignados se derivan de las calificaciones cuantitativas para las cuales asignamos una nota determinada. Así:

- Muy bueno (MB), si la asignación vigesimal es 18, 19 o 20.

- Bueno (B) equivale a 15, 16 o 17.

- Regular (R) si la calificación alcanza 12, 13 o 14.

- Deficiente (D) si le corresponde una nota de 09, 10 o 11.

- Muy deficiente (MD) si la calificación es menor a 08.

Tabla 2

Calificación valorativa sobre el uso de los mecanismos de cohesión descritos. Universidad Nacional de Ingeniería, Lima, Perú

\begin{tabular}{|c|c|c|c|c|c|c|}
\hline $\mathrm{N}^{\circ}$ & \multicolumn{3}{|c|}{ Grupo de control } & \multicolumn{3}{|c|}{ Grupo cuasi experimental } \\
\hline & $\mathrm{I}$ & II & III & I & II & III \\
\hline 1 & D & $\mathrm{R}$ & D & MB & B & $\mathrm{MB}$ \\
\hline 2 & $\mathrm{R}$ & $\mathrm{D}$ & B & B & B & B \\
\hline 3 & $\mathrm{D}$ & D & $\mathrm{D}$ & $\mathrm{R}$ & $\mathrm{B}$ & $B$ \\
\hline 4 & $\mathrm{R}$ & $\mathrm{R}$ & B & $\mathrm{B}$ & B & MB \\
\hline 5 & $\mathrm{R}$ & $\mathrm{R}$ & $B$ & B & $B$ & $B$ \\
\hline 6 & $\mathrm{~B}$ & $\mathrm{R}$ & B & $\mathrm{R}$ & $\mathrm{R}$ & $B$ \\
\hline 7 & MD & MD & MD & B & MB & MB \\
\hline 8 & $\mathrm{R}$ & $\mathrm{R}$ & $\mathrm{R}$ & $\mathrm{MB}$ & $\mathrm{MB}$ & $\mathrm{MB}$ \\
\hline 9 & $\mathrm{D}$ & $\mathrm{D}$ & $\mathrm{R}$ & $\mathrm{R}$ & $\mathrm{R}$ & $\mathrm{B}$ \\
\hline 10 & D & D & $\mathrm{R}$ & $\mathrm{B}$ & B & $B$ \\
\hline 11 & $\mathrm{R}$ & $\mathrm{R}$ & MD & $\mathrm{B}$ & $\mathrm{B}$ & $\mathrm{B}$ \\
\hline 12 & $\mathrm{R}$ & $\mathrm{D}$ & $\mathrm{D}$ & MB & $B$ & MB \\
\hline 13 & D & $\mathrm{R}$ & $\mathrm{R}$ & $\mathrm{R}$ & $\mathrm{R}$ & $\mathrm{R}$ \\
\hline 14 & $\mathrm{R}$ & $B$ & $B$ & $\mathrm{R}$ & $\mathrm{MB}$ & MB \\
\hline 15 & $\mathrm{D}$ & $D$ & $\mathrm{R}$ & $\mathrm{B}$ & $\mathrm{R}$ & $\mathrm{B}$ \\
\hline 16 & D & D & $\mathrm{D}$ & $B$ & B & $B$ \\
\hline 17 & MD & $\mathrm{D}$ & $\mathrm{R}$ & $B$ & $B$ & $\mathrm{R}$ \\
\hline 18 & $\mathrm{R}$ & $\mathrm{B}$ & $B$ & $\mathrm{~B}$ & $\mathrm{~B}$ & $\mathrm{~B}$ \\
\hline 19 & MD & $\mathrm{R}$ & $\mathrm{R}$ & $B$ & MB & $\mathrm{MB}$ \\
\hline 20 & $\mathrm{R}$ & B & $B$ & B & B & B \\
\hline 21 & D & $\mathrm{D}$ & $\mathrm{R}$ & MB & MB & MB \\
\hline 22 & $\mathrm{R}$ & $\mathrm{R}$ & $\mathrm{R}$ & $B$ & $B$ & $\mathrm{R}$ \\
\hline 23 & $B$ & $B$ & B & $\mathrm{R}$ & $\mathrm{R}$ & $\mathrm{R}$ \\
\hline 24 & $\mathrm{MD}$ & $\mathrm{D}$ & $\mathrm{R}$ & $B$ & $B$ & $B$ \\
\hline 25 & $\mathrm{R}$ & $\mathrm{R}$ & $\mathrm{R}$ & $\mathrm{MB}$ & $\mathrm{B}$ & MB \\
\hline
\end{tabular}

Fuente: Elaboración propia. (2017). 


\subsection{Resultados de la prueba del grupo de control}

Para caracterizar la redacción de los integrantes del grupo de control, vamos a tomar como base el siguiente texto:

\section{Texto 1}

El tema de la contaminación ambiental hoy en día es un tema muy discutido por la prensa y distintas personas, que critica muy duramente al gobierno puesto que este con tal de generarse más ingresos y poder mejorar el PIB sacrifican algunas provincias y localidades que resultan perjudiciales, perjudicando principalmente a la tierra que en algunos casos le puede causar daños irreparables.

La contaminación ambiental es un tema muy amplio que abarca diversos temas de contaminación, como es el caso en el Perü, que existe principalmente una contaminación de tipo minera, puesto que el Perü recibe diversas empresas extranjeras que lo principal que hacen es explotar las tierras que tienen en su poder, sin importarle perjudicar a los ciudadanos que habitan en esta, arrojando sus relaves mineros a los ríos sin importarles volverlos tóxicos, algo que el estado permite, un claro ejemplo: podríamos poner el caso de Conga en el cual la provincia de Cajamarca (donde se desarrollará el proyecto) se opone a su ejecución, puesto que el proyecto conllevará a la contaminación siendo los principales afectados los pobladores. Otro tipo de contaminación ambiental podría ser la contaminación con respecto al aire que es muy tocado hoy en día puesto que se está destruyendo la capa de ozono, que podríamos evitar contaminando menos como por ejemplo evitar el uso de aerosoles, químicos entre otros.

La conclusión sería que el estado peruand pueda dejar trabajar a las empresas extranjeras puesto que generan ingresos, siempre y cuando el estado establezca normas extrictas a las empresas, pues deberían de cumplir al extrecho margen y para que así se pueda beneficiar al estado y también no salgan perjudicados los habitantes, puesto que la principal función del gobierno es velar por el bienestar de ellos, así de esta forma que salgan ganando todas las partes para que así el Perú progrese y podamos ganar todos, beneficiándonos de esto. 
La primera impresión que nos causa este texto es su estructura sintáctica intrincada y la extensión de las oraciones. A pesar de contener trescientas once palabras, el texto está segmentado en cuatro oraciones solamente. Esto implica que el número de palabras para cada oración es de setenta y cinco términos, aproximadamente. Este número de palabras en una sola oración concibe que la información contenida en el texto no fluye de manera ordenada, clara ni lógica. Antes bien, las ideas resultan confusas y enrevesadas. En otros términos, el productor textual no controla estratégicamente la función de los elementos cohesivos, por lo que las ideas expresadas se dispersan generando problemas para su debida comprensión. En consecuencia, podemos señalar algunos aspectos centrales de los textos evaluados.

1. Las repeticiones no están adecuadamente establecidas para dinamizar la progresión temática. Este hecho conlleva a contar con un texto, colmado de redundancias.

2. Se advierte sustituciones imprecisas en el interior de los enunciados, situación que no solo genera discordancias gramaticales, sino que, además, sesga la progresión temática en los textos.

3. Vemos pocos elementos cohesivos al interior de los textos. Además, en algunos casos, no concatena la relación entre la información conocida y la información nueva, es decir, no hay clara distinción entre tema-rema.

4. El redactor no advierte que los verbos en español contienen de manera implícita morfemas de persona, número, tiempo, modo y aspecto, por lo que sería recomendable recurrir a esta estrategia.

5. Si bien se advierte la presencia de conectores textuales; en muchos casos, no son utilizados de manera adecuada ni precisa.

\subsection{Resultados de la investigación cuasi experimental}

Para explicar los resultados obtenidos en el grupo cuasi experimental, tomamos en cuenta el texto 2 , escrito por un integrante de este grupo. 


\section{Texto 2}

(1) Cuando hablamos de contaminación ambiental, nos referimos a una combinación de varios agentes nocivos en el medio $\varnothing$. (2) Estos agentes pueden ser tanto físicos, químicos como biológicos. (3) Todo ello afecta a los seres humanos, animales y plantas.

(4)Actualmente, la contaminación ambiental es un problema de carácter mundial y uno de sus componentes más resaltantes es la explotación minera. (5)La minería genera partículas que alteran el medio ambiente y además $\varnothing$ atentan contra la vida. (6)En el Perú, la contaminación ambiental está relacionada con la extracción indiscriminada de los recursos minerales. (7)En esta actividad intervienen muchas compañías mineras extranjeras, pero también $\varnothing$ las minerías informales, generando los relaves mineros. (8)Los relaves mineros son sustancias tóxicas que atentan contra la vida. (9) Estos desechos son expulsados al medio, generando graves daños en el ecosistema local. (10)Entre los daños que ocasionan son: agua no apta para el consumo, tierras infértiles, pérdida de cosechas. (11)La Oroya es un caso extremo de esta realidad, pues los desechos tóxicos son arrojados al río Mantaro y sus aguas no sirven para el consumo.

(12)En conclusión, la contaminación ambiental es uno de los problemas más álgidos hoy en día, porque $\varnothing$ afecta a todo el planeta. (13)En la actualidad, la actividad que genera mayor contaminación es la minería. (14)En países de buena tecnología y buenas costumbres esto no sucede. (15)Por eso como país tenemos que crear leyes que regulen las actividades mineras. (16)El gobierno debe incentivar una minería responsable. (17) Una minera responsable es aquella que cumple los parámetros ambientales. (18)Sobre todo es aquella que respeta el medio ambiente.

\subsection{Uso de los mecanismos de cohesión textual investigados}

A continuación, pasamos a explicar algunos rasgos que caracterizan los textos elaborados por los integrantes de este grupo. Para ello, tomamos en cuenta solo el uso de la repetición, la sustitución y la elisión.

\subsubsection{Uso de la Repetición}

En los textos observados, la repetición consiste en la sola reiteración de palabras o expresiones para designar el mismo referente. La repetición puede ser total o parcial. La 
primera es uno de los procedimientos de cohesión más sencillos que se advierte en los textos. La segunda, más bien, transcategoriza un elemento ya utilizado con anterioridad en otro tipo de elemento, como vemos en el siguiente texto.

(1)Cuando hablamos de contaminación ambiental, nos referimos a una combinación de varios agentes nocivos en el medio. (2)Estos agentes pueden ser tanto físicos, químicos como biológicos.

En este texto, la expresión agente nocivo de la oración 1 se repite en el siguiente enunciado, pero solo como agentes y no figura ya el término nocivo.

Ahora bien, ¿cuáles son las limitaciones de la repetición como mecanismo de cohesión? Si bien, la repetición es una estrategia cohesiva muy productiva en la redacción, este recurso también puede reflejar la pobreza léxica por parte del productor textual. Si el redactor repite la misma palabra, podemos inferir que utiliza el mismo término sin percatarse de ello. También, podemos advertir que el autor no cuenta con otra expresión para sustituirla, tal como se advierte en el siguiente texto:

"El tema de la contaminación ambiental hoy en día es un tema muy discutido por la prensa y distintas personas..."

Aquí, la expresión tema se reproduce en dos ocasiones y hace alusión al mismo referente, pero el segundo elemento no aporta información adicional. Así, en periodos relativamente breves, este mecanismo no resulta pertinente para generar una cohesión adecuada al texto.

\subsubsection{Comparación}

Para comparar los resultados, mostramos en la Tabla 3, las tendencias en el uso de la repetición como mecanismo de cohesión textual tanto del grupo de control como del grupo cuasi experimental. 
Tabla 3

Frecuencia y porcentaje de estudiantes por calificación en la visualización de la repetición como mecanismo de cohesión, según grupo control y cuasi experimental, Universidad Nacional de Ingeniería, Lima-Perú.

\begin{tabular}{|c|c|c|c|c|}
\hline \multicolumn{5}{|c|}{ Repetición } \\
\hline Calificación & \multicolumn{2}{|c|}{ Grupo de control } & \multicolumn{2}{|c|}{$\begin{array}{l}\text { Grupo cuasi } \\
\text { experimental }\end{array}$} \\
\hline & Frecuencia & Porcentaje & Frecuencia & Porcentaje \\
\hline MB & 0 & 0 & 5 & 20 \\
\hline B & 2 & 8 & 14 & 56 \\
\hline $\mathrm{R}$ & 11 & 44 & 6 & 24 \\
\hline D & 8 & 32 & 0 & 0 \\
\hline MD & 4 & 16 & 0 & 0 \\
\hline Total & 25 & 100 & 25 & 100 \\
\hline
\end{tabular}

Fuente: Elaboración propia. (2017).

a. Grupo de control. De la totalidad de los 25 textos redactados por los integrantes del grupo de control, la repetición se presenta del siguiente modo: MB: 0\%, B: 8\%, R: 44\%, D: $32 \%$, MD: 16\%. Como se advierte, hay una tendencia pobre en el manejo de la repetición como mecanismo de cohesión.

b. Grupo cuasi experimental. En este grupo, el porcentaje de repeticiones se presenta como sigue: $\mathrm{MB}: 20 \%$, B: $56 \%$, R: $24 \%$, D: 0\%, MD: 0\%.

Estas diferencias parciales reflejan que los integrantes del grupo cuasi experimental (en comparación con los del grupo de control) se orientan hacia el manejo cada vez más consistente y eficaz de la repetición. En este sentido, la repetición, a primera vista, pareciera generar redundancia al texto; sin embargo, si la utilizamos con propiedad, esta puede ser no solo útil, sino necesaria como elemento cohesivo. Ello no sucede en el grupo de control. Aquí se advierte la tendencia hacia el manejo no apropiado y/o el uso de las repeticiones superfluas en el contexto en el que aparecen. Desde luego, la tendencia hacia el uso más apropiado de este mecanismo por parte del grupo cuasi experimental podría deberse a la eficacia de la sintaxis en la redacción. Esta apreciación es tentativa, pues posteriormente la compararemos con los resultados del uso de otros mecanismos de cohesión textual. Se debe aclarar que estas diferencias se dan en puntos porcentuales. 


\subsubsection{Uso de la Sustitución}

En los textos escritos, la persona discente recurre a este elemento cohesivo para evitar así la repetición de un mismo término al interior del párrafo. La sustitución puede darse mediante una sinonimia léxica o por medio de pronombres, tal como se muestra en el siguiente texto:

(1)En el Perú, la contaminación ambiental está relacionada con la extracción indiscriminada de los recursos minerales. (2)En esta actividad intervienen muchas compañías mineras extranjeras.

Aquí, se recurre a la expresión esta actividad para sustituir la frase extracción indiscriminada de los recursos minerales. Si bien, esta sustitución no aporta información novedosa, dinamiza el desarrollo de la progresión temática haciendo que las ideas en el texto no fluyan de manera dinámica. Ahora, ¿cuáles son las dificultades para llevar a cabo sustituciones adecuadas? Estas dificultades están relacionadas con el bajo nivel de la competencia lexical del redactor. Para ello, es imprescindible afianzar dicha competencia y, de ese modo, contar con un bagaje lexical sólido. Gracias a esta competencia podemos seleccionar términos puntuales según el contexto en el que debe incluirse el término sustituto. Respecto de los pronombres, es necesario controlar las concordancias gramaticales de género y número para evitar fenómenos como loísmo, laísmo, leísmo, temas trillados sobre las incorrecciones gramaticales en la redacción.

\subsubsection{Comparación}

Para comparar los resultados, observemos la Tabla 4.

\section{Tabla 4}

Frecuencia y porcentaje de estudiantes por calificación en la visualización de la sustitución como mecanismo de cohesión, según grupo control y cuasi experimental, Universidad Nacional de Ingeniería, Lima-Perú

\begin{tabular}{|l|r|r|r|r|}
\hline \multicolumn{5}{|c|}{ SUSTITUCIÓN } \\
\hline Calificación & \multicolumn{2}{|c|}{ Grupo de control } & \multicolumn{2}{c|}{ Grupo cuasi experimental } \\
\hline & Frecuencia & Porcentaje & Frecuencia & Porcentaje \\
\hline MB & 0 & 0 & 5 & 20 \\
\hline B & 4 & 16 & 15 & 60 \\
\hline R & 10 & 40 & 5 & 20 \\
\hline D & 10 & 40 & 0 & 0 \\
\hline MD & 1 & 4 & 0 & 0 \\
\hline Total & $\mathbf{2 5}$ & $\mathbf{1 0 0}$ & $\mathbf{2 5}$ & $\mathbf{1 0 0}$ \\
\hline
\end{tabular}

Fuente: Elaboración propia (2017). 
Ahora comparemos las frecuencias en el uso de este elemento cohesivo.

a. Grupo de control. En los integrantes del grupo de control, sin considerar específicamente la clase de palabra o expresión, la tendencia es la siguiente: MB: 00\%, B: $16 \%$, R: $40 \%$, D: $40 \%$, MD: $4 \%$.

b. Grupo cuasi experimental. En este grupo, las sustituciones se dan del siguiente modo: MB: $20 \%$, B: $60 \%$, R: $20 \%$, D: $00 \%$, MD: $00 \%$.

La comparación de estos resultados, que son a nivel porcentual, nos permite advertir que el uso de este mecanismo en el grupo de control es deficiente. En cambio, en el grupo cuasi experimental, hay una propensión marcada hacia el uso apropiado de este mecanismo. Así pues, en el grupo cuasi experimental, hay un manejo proporcionado y consistente de la sustitución. En el grupo de control, vemos el uso irregular de este elemento cohesivo.

\subsubsection{Uso de la Elisión}

Para comentar el uso de este mecanismo de cohesión, veamos el siguiente texto:

La minería altera el medio ambiente y, además, atenta contra la vida.

En este caso, en la segunda proposición, se omite la expresión la minería que viene a ser el sujeto de la segunda cláusula. En la producción de textos, la elisión está basada en el principio de economía lingüística. En este caso, si la repetición le otorga seguridad a la coherencia textual, la omisión le confiere dinamismo comunicativo. El siguiente texto, corrobora lo señalado aquí. Veamos:

(7)En esta actividad intervienen muchas compañías mineras extranjeras, pero también $\varnothing$ las minerías informales, generando los relaves mineros.

Aquí, la parte elidida (Ø), es decir intervienen, no resulta imprescindible para que el texto tenga sentido. Antes bien, genera dinamismo comunicativo. En este caso, el redactor, al suprimir esas ideas, presume que el receptor ya conoce dicha información y confía en que la puede identificar por el contexto. Sin embargo, ¿qué ocurre si llevamos a cabo elisiones desmedidas? Algunas veces, el redactor puede caer en el otro fenómeno llamado texto telegráfico. En este caso, omite más información de lo necesario, por lo que el lector debe realizar mayor inferencia para recuperar la información suprimida. Al respecto, veamos el siguiente texto. 
El medio ambiente es un sistema natural que, en un estado inicial, se encuentra en equilibrio. El equilibrio se ve afectado por diversos agentes, los cuales son originados principalmente por el hombre.

El tema del texto está referido a la 'contaminación ambiental', pero el lector requiere mayor información para saber que ese párrafo desarrolla ese tema. Por eso, en lugar de suprimir más información, el redactor tendría que explicitarlas cuando estas sean necesarias.

\subsubsection{Comparación}

Para explicar las características respecto del uso de este recurso cohesivo, observemos la Tabla 5.

\section{Tabla 5}

Frecuencia y porcentaje de estudiantes por calificación en la visualización de la elisión como mecanismo de cohesión, según grupo control y cuasi experimental, Universidad Nacional de Ingeniería, Lima-Perú

\begin{tabular}{|l|r|r|r|r|}
\hline \multicolumn{5}{|c|}{ ELISIÓN } \\
\hline Calificación & \multicolumn{2}{|c|}{ Grupo de control } & \multicolumn{2}{c|}{ Grupo cuasi experimental } \\
\hline & Frecuencia & Porcentaje & Frecuencia & Porcentaje \\
\hline MB & 0 & 0 & 09 & 36 \\
\hline B & 8 & 32 & 12 & 48 \\
\hline R & 11 & 44 & 4 & 16 \\
\hline D & 4 & 16 & 0 & 0 \\
\hline MD & 2 & 8 & 0 & 0 \\
\hline Total & $\mathbf{2 5}$ & $\mathbf{1 0 0}$ & $\mathbf{2 5}$ & $\mathbf{1 0 0}$ \\
\hline
\end{tabular}

Fuente: Elaboración propia (2017).

Si bien los porcentajes muestran algunas tendencias, es necesario comparar para ver las similitudes y las diferencias.

a. Grupo de control. En los integrantes de este grupo, sin incluir específicamente la clase de palabra o expresión que se omite, la tendencia es la siguiente: MB: 00\%, B: $32 \%, \mathrm{R}$ : 44\%, D: $16 \%$, MD: $8 \%$

b. Grupo cuasi experimental. En este grupo, tenemos los siguientes resultados: MB: 36\%, B: 48\%, R: 16\%, D: 0\%, MD: 00\%. Como vemos, el uso de este mecanismo es consistente si consideramos que la redacción académica debe garantizar un texto adecuado y eficaz. 
Así pues, el grupo cuasi experimental utiliza este elemento cohesivo con mayor propiedad. Esto es, si la elisión le permite proveer concisión y precisión al texto; entonces, el redactor que conoce la función de esta estrategia recurrirá a este elemento para dar cohesión al texto. Sin embargo, en materia de redacción académica, no todo es color de rosa. El uso recurrente de los elementos como la repetición, la sustitución, la elisión y otros mecanismos de reiteración, también, puede generar problemas al texto si estos elementos aparecen en periodos breves al interior de los párrafos.

\subsection{Implicaciones de los elementos de cohesión en la coherencia del texto}

Como sabemos, un texto es coherente si su estructura significativa tiene organización lógica, armonía sintáctica, relación semántica y pragmática entre sus partes. En otras palabras, la coherencia de un texto es una propiedad de la sustancia textual que, a nivel de la estructural sintáctica, se expresa mediante un conjunto de mecanismos lingüísticos de cohesión. En este sentido, para que un texto resulte coherente entre sus elementos, deben intervenir, de forma proporcional, elementos cohesivos como la repetición, la sustitución, la elisión, las proformas, los conectores textuales, entre otros. Estos elementos van a generar la coherencia lineal y global del texto, tal como se muestra en el siguiente ejemplo:

(1) Cuando hablamos de contaminación ambiental, nos referimos a una combinación de varios agentes nocivos en el medio. (2) Estos agentes pueden ser tanto físicos, químicos como biológicos. (3) Todo ello afecta a los seres humanos, animales y plantas.

a. La coherencia lineal. Para formar la coherencia lineal, los segmentos 1,2 y 3 se cohesionan. Es decir, la relación semántica se da entre cláusula y cláusula generando una coherencia lineal. Para ello, intervienen los elementos cohesivos como las repeticiones, las sustituciones y las elisiones.

b. La coherencia global. Constituye la unidad temática del texto. Esta unidad se establece a partir del tema central que desarrolla el texto y sus relaciones con las distintas partes del todo. En este ejemplo, el texto gira en torno al tema referido a la contaminación ambiental.

En concordancia con lo señalado hasta aquí, diremos que la implementación de la estrategia metodológica propuesta ofrece ventajas para afianzar la competencia textual y discursiva de los discentes. 


\section{Conclusiones}

A continuación, daremos las conclusiones a las que hemos llegado.

1. Cuando la muestra cuasi experimental elabora materiales escritos, sus integrantes redactan textos bastante cohesionados. Para tal efecto, utilizan oraciones breves y con la presencia constante de elementos cohesivos para concatenar las ideas. Los integrantes del grupo de control utilizan, generalmente, oraciones extensas. Por ello, los textos redactados resultan intrincados y no puntualizan, adecuadamente, el tema que desarrollan.

2. La muestra que representa el grupo cuasi experimental utiliza la repetición de manera proporcional para dinamizar la progresión temática. De este modo, los textos que redactan resultan ágiles y fluidos para su lectura. En la redacción de los integrantes del grupo de control, si bien se advierte la presencia de este mecanismo; sin embargo, el uso de este elemento no es regular ni planificado, situación que conlleva al deterioro de la calidad del texto.

3. Las personas discentes que forman el grupo cuasi experimental, de manera constante, recurren al uso de la sustitución como mecanismo de cohesión. Esta sustitución se presenta, preferentemente, mediante la sinonimia y los pronombres. Al recurrir al uso de este elemento, la sintaxis del texto resulta ágil y dinámica. En los participantes del grupo de control, si bien se advierte la presencia de algunas sustituciones, su uso no es constante ni tampoco se visualiza como una estrategia planificada.

4. Las personas discentes que integran el grupo cuasi experimental recurren, sobremanera, a las elisiones de algunos elementos de las oraciones cuando componen los textos. Así, por ejemplo, cuando recurren a los verbos conjugados, obvian, en muchos casos, el sujeto de las oraciones. De este modo, la progresión temática de los textos muestra fluidez entre sus componentes. En la redacción de los integrantes del grupo de control, también se advierte las elisiones, aunque no utilizan de manera planificada este recurso.

Así pues, respecto de la competencia discursiva que han alcanzado los integrantes de la muestra cuasi experimental, podemos precisar que esa forma de redactar se aproxima a las características sintácticas de los textos científicos. Cuando observamos la estructura discursiva que poseen textos de Física, Química, Geometría, Biología advertimos que la 
sintaxis de esta clase de materiales resulta sencilla y llana, donde los elementos cohesivos como la repetición, la sustitución, las elisiones, las proformas, las paráfrasis y los tiempos verbales son constantes para enlazar las secuencias informativas. Si esta clase de textos posee estas características; entonces, la orientación estratégica que proponemos no solo resultará viable, sino que será eficaz para afianzar la competencia discursiva de la población estudiantil perteneciente a las áreas no humanísticas como la Arquitectura y la Ingeniería.

\section{Referencias}

Beaugrande, Robert y Dressler, Wolfgang. (1997). Introducción a la Lingüística del texto. Barcelona: Ariel.

Bernárdez, Enrique. (1982). Introducción a la Lingüística del texto. Madrid: ESPASA-CALPE.

Bernárdez, Enrique. (1995). Teoría y epistemología del texto. Barcelona: Cátedra.

Bosque, Ignacio y Gutiérrez, Javier. (2009). Fundamentos de sintaxis formal. Madrid: Akal.

Calsamiglia, Helena y Tusón, Amparo. (2016). Las cosas del decir. Barcelona: Ariel.

Carlino, Paula. ((2005a). Escribir, leer, y aprender en la universidad. Una introducción a la alfabetización académica. Buenos Aires, F.C.E. Recuperado de https://www.aacademica.org/paula.carlino/3.pdf.

Carlino, Paula. (2005b). La escritura en el nivel superior. Recuperado de https://pesmartinezsin.infd.edu.ar/sitio/curso-de-ingreso-

2018/upload/La escritura en el nivel superior.Doc2.pdf

Carlino, Paula. (2013). Alfabetización académica diez años después. En Revista mexicana de investigación educativa. RMIE, vol.18 no.57 México abr./jun. Recuperado de http://www.scielo.org.mx/scielo.php?pid=S1405$66662013000200003 \&$ script=sci arttext\&tlng=en

Cassany, Daniel y Morales, Óscar. (2008). "Leer y escribir en la universidad: Hacia la lectura y la escritura crítica de géneros científicos". En Revista Memoralia, Universidad Nacional Experimental de los Llanos Ezequiel Zamora (Unellez), Cojedes, Venezuela. En proceso de publicación. Recuperado de http://www.saber.ula.ve/bitstream/handle/123456789/16457/leer universidad.pdf;jsessi onid=A377C1B19A8BC799D71CD79A43C9D4BB? sequence $=1$.

Coseriu, Eugenio. (2007). Lingüística del texto. Madrid: Arco/Libros.

Gutiérrez, Salvador. (2002). De pragmática y semántica. Madrid: Arco/Libros.

Regueiro, María y Sáez, Daniel. (2015). El español académico. Madrid: Arco/Libros.

Van Dijk, Teun. (1983). La ciencia del texto. Barcelona: Paidós. Madrid: Arco/Libros. 
Revista indizada en
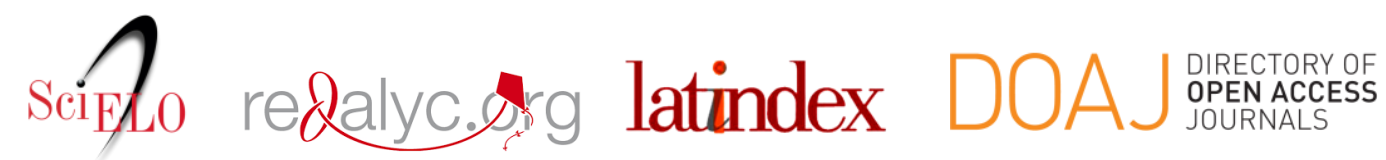

Distribuida en las bases de datos:

- Dialnet

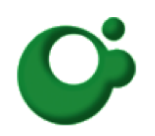
SHERPA/RoMEO REDIB

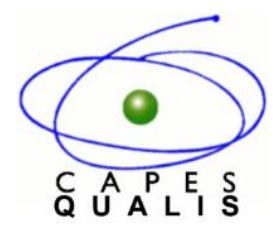

MIAR 